\title{
The effects of reflex path length on clonus frequency in spastic muscles
}

\author{
ROBERT IANSEK \\ From the Department of Neurology, Prince Henry's Hospital, Melbourne, Australia
}

SUMMARY Clinical evaluation of clonus in spastic muscles was performed by comparing reflex path length with clonus frequency for different muscles in the same patient. It was found that clonus frequency varied inversely with reflex path length $(r=0.84, p<0.001)$. The findings confirm that clonus is generated by a peripheral mechanism of self re-excitation rather than a central spinal pacemaker.

Clonus is a well recognised clinical sign, usually occurring in spasticity. A normal, nonspastic limb may manifest clonus, but only under certain conditions.' The repetitive self perpetuating movement is induced by brisk stretch of the involved muscle group, although cutaneous sensory inputs can initiate clonic movements in a susceptible limb. ${ }^{2}$ The underlying mechanism of clonus is poorly understood and controversial. Some investigators ${ }^{34}$ have evidence to support the theory of a self re-excitation circuit perpetuated by the constant stretch applied to the contracting muscle, on a background of high gain in the motor neuron-spindle feed back loop. Other investigators, ${ }^{25}$ claim that the evidence supports the concept of a spinal pacemaker which has been released by lesions to descending motor pathways and that abrupt spindle input, induced by mechanical muscle stretch, switches on the spinal pacemaker to produce the clonus.

This report outlines the results of an investigation which tested the relationship of clonus frequency with the distance of the contracting muscle from the spinal cord. The results clearly show that a linear inverse relationship does exist between clonus frequency and reflex path length. It is concluded that such a finding provided further support for a peripheral self re-excitation mechanism, rather than an intrinsic spinal pacemaker in the mechanism of clonus.

Address for reprint requests: Dr R Iansek, Department of Neurology, Middlesex Hospital, London W1N 8AA, UK.

Received 31 January 1984 and in revised form 5 April 1984. Accepted 18 April 1984

\section{Methods}

Twenty-one unselected patients who were seen on the neurology service were used as subjects. Attempts were $\bar{O}$ made to study patients with clonus in more than one mus-疋 cle. No restriction was placed on the underlying pathologi- $\overrightarrow{\mathbb{D}}$ cal basis for the spasticity. Neurological examination and $\mathrm{Q}$ routine nerve conduction studies were performed to exclude an underlying neuropathy. Clonus frequency was measured by use of surface electrodes and the raw EMGO was recorded on photosensitive paper. The duration of each EMG burst was halved and the mid point was used to estimate the interbeat time for ten successive EMG bursts and then averaged to obtain the frequency. The approximate distance of the clonic muscles from the spinal cord was calculated as follows:

(1) Ankle The distance from the medial malleolus to the anterior superior iliac spine.

(2) Knee The anterior tibial plateau to the anterior superior iliac spine.

(3) Forearm The lateral epicondyle to C7 vertebral spine with the limb in the anatomical position.

\section{Results}

The table summarises the results for all 21 patients. Twelve patients (19 recordings) had full nerve conduction velocity estimation, whereas the remainder (9) refused to proceed with further analysis subsequent to the estimation of the distal motor latency because of discomfort. No patient had either clinical or electrical evidence of a peripheral neuropathy. The probable pathological basis for the spasticity was quite variable, as was the age. The results were analysed according to which patients had had a complete and incomplete nerve conduction velocity 
Table Summary of results

\begin{tabular}{|c|c|c|c|c|c|c|c|}
\hline \multirow{2}{*}{$\begin{array}{l}\text { Patient } \\
\text { Age (yr) }\end{array}$} & \multirow[t]{2}{*}{ Provisional diagnosis } & \multicolumn{2}{|l|}{ Leg } & \multicolumn{2}{|c|}{ Thigh } & \multicolumn{2}{|c|}{ Arm } \\
\hline & & $\mathrm{Hz}$ & $C M S$ & $H z$ & $C M S$ & $H z$ & $C M S$ \\
\hline $\begin{array}{l}41 \\
23 \\
50 \\
71 \\
56 \\
46 \\
23 \\
50 \\
33 \\
26 \\
56 \\
54 \\
22 \\
75 \\
75 \\
68 \\
58 \\
81 \\
28 \\
39 \\
16\end{array}$ & $\begin{array}{l}\text { Multiple sclerosis } \\
\text { Familial spastic paraparesis } \\
\text { Stroke } \\
\text { Stroke } \\
\text { Stroke } \\
\text { Multiple sclerosis } \\
\text { Unknown } \\
\text { Multiple sclerosis } \\
\text { Cerebral cysticercosis } \\
\text { Pseudobulbar palsy } \\
\text { Multiple sclerosis } \\
\text { Unknown } \\
\text { Motor vehicle accident, head injury } \\
\text { Cervical cord compression } \\
\text { Brainstem stroke } \\
\text { Cervical cord compression } \\
\text { Vitamin B12 deficiency } \\
\text { Cervical cord compression } \\
\text { Syringomyelia } \\
\text { Multiple sclerosis } \\
\text { Herpes simplex encephalitis }\end{array}$ & $\begin{array}{l}6 \cdot 5 \\
7 \cdot 2 \\
6 \cdot 7 \\
6 \cdot 1 \\
6 \cdot 3 \\
8 \cdot 0 \\
5 \cdot 9 \\
6 \cdot 5 \\
6 \cdot 7 \\
7 \cdot 0 \\
5 \cdot 7 \\
6 \cdot 9 \\
6 \cdot 5 \\
5 \cdot 9 \\
5 \cdot 7 \\
5 \cdot 0 \\
7 \cdot 0 \\
4 \cdot 4 \\
6 \cdot 0 \\
6 \cdot 9 \\
7 \cdot 7\end{array}$ & $\begin{array}{l}85 \\
91 \\
90 \\
90 \\
84 \\
77 \\
88 \\
78 \\
82 \\
88 \\
83 \\
83 \\
85 \\
84 \\
81 \\
89 \\
78 \\
93 \\
95 \\
83 \\
82\end{array}$ & $\begin{array}{l}- \\
- \\
- \\
- \\
- \\
- \\
\overline{7} \\
-5 \\
- \\
\overline{10} \\
7 \cdot 8 \\
- \\
- \\
- \\
- \\
-\end{array}$ & $\begin{array}{l}- \\
- \\
- \\
- \\
- \\
\overline{54} \\
- \\
- \\
54 \\
54 \\
- \\
- \\
- \\
-\end{array}$ & $\begin{array}{l}- \\
\overline{9 \cdot 5} \\
\overline{8} \\
\overline{7 \cdot 6} \\
9 \cdot 3 \\
- \\
\overline{8 \cdot 3} \\
- \\
- \\
- \\
- \\
- \\
- \\
-\end{array}$ & $\begin{array}{l}- \\
\overline{53} \\
\overline{64} \\
\overline{54} \\
48 \\
\overline{-} \\
\overline{47} \\
- \\
- \\
- \\
- \\
- \\
-\end{array}$ \\
\hline
\end{tabular}

estimation. The plot of the clonus frequency against approximate distance from the spinal cord is shown in the figure for both groups of patient. Results of linear regression analysis are also shown in the figures for each group in the form of the line of best fit and its confidence limits. The correlation coefficient for the group of patients in which nerve conduction velocity was estimated was -0.84 ( $p<$ 0.001 ) and for the total number of patients the correlation coefficient was $-0 \cdot 80(p<0.001)$. It is evidence that a linear relationship existed between the clonus frequency and the distance of the muscle

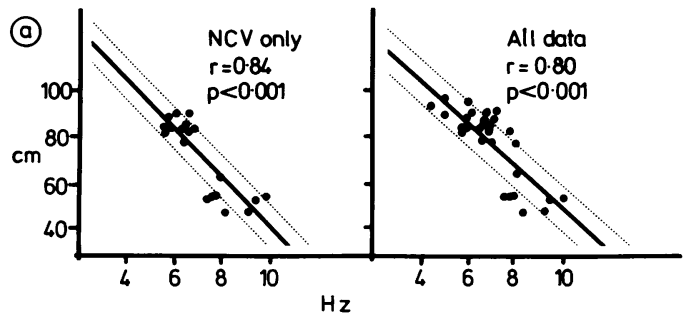

(b)

Ankle $5 \cdot 7 \mathrm{~Hz}$

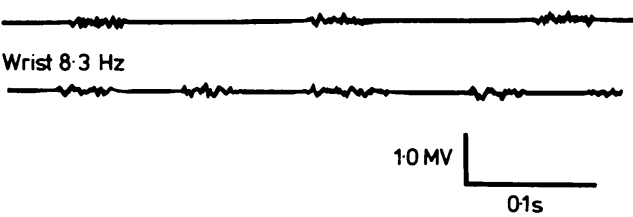

Fig (a) Regression plot for reflex path length (CMS) and clonus frequency $(\mathrm{Hz})$. NCV-nerve conduction velocity estimated and normal in the patients used in the plot. (b) Raw EMG comparison of ankle and wrist clonus in same patient with multiple sclerosis. from the spinal cord. An example of this finding is shown in figure 1(b) where the raw EMG activity of the gastrocnemius muscle for clonus occurring at the ankle is compared with the EMG activity of the muscle producing clonus at the wrist. In another patient with widespread clonus and a pseudobulbar palsy of uncertain cause, several clonic beats could be induced by the performance of a jaw jerk. The frequency in this case was $13.8 \mathrm{~Hz}$ compared to a clonic frequency of $7.0 \mathrm{~Hz}$ at the ankle. Although, this was not sustained clonus the finding is in agreement with the remainder of this report, as one would predict that clonus of the jaw would be at a much faster frequency than at the ankle.

\section{Discussion}

Clonus frequency is well known to vary over a narrow range. However, it has never been shown that within that narrow range the frequency is dependent linearly on the length of the reflex loop. Mathematically, reflex oscillation latency is the predominant determinant of frequency. ${ }^{\circ}$ A central spinal pacemaker could not explain the current findings as such a theory would predict the same clonic frequency irrespective of the reflex path length for the same individual, given that the pathology was consistent for the respective clonic muscles. Rather, the findings are in agreement with a purely peripheral self re-excitation mechanism, mediated by a high gain reflex loop.

Walsh, ${ }^{5}$ and Dimitrijevic, Nathan and Sherwood, ${ }^{2}$ have been strong proponents for a central pacemaker mechanism. Walsh ${ }^{5}$ based his conclusion on the fact that he could not entrain the clonus fre- 
quency of spastic patients with an applied sinusoidal force of a set frequency, but rather that a beat frequency was set up. Such a finding, he concluded, was consistent with a central mechanism. However, Stein and Lee, ${ }^{6}$ claim that beat frequencies can occur purely on a simple peripheral oscillation reflex, rather than on a central mechanism and have proved this point mathematically. Dimitrijevic, Nathan and Sherwood ${ }^{2}$ based their conclusions on the fact that clonic motor neurons were refractory to sensory inputs from the $1 \mathrm{~A}$ fibres subsequent to a clonic contraction for a set period which coincided with the clonus frequency. Furthermore, they were unable to change the frequency by a variety of manoeuvres which would theoretically alter the frequency if the mechanism was a peripheral one. The inexcitability of the motor neuron pool in the Dimitrijevic, Nathan and Sherwood study ${ }^{2}$ was short enough to put a limit on the higher frequency, but not on the lower frequency of clonus. The latter would be determined by reflex delay times. In addition, none of the manoeuvres used in that study were assessed for their effect on the peripheral reflex pathways; an example is the effect of temperature change on conduction velocity.

One criticism of the current study is that the relationship between reflex path length and frequency might be artefactual owing to an inaccurate measurement of reflex path lengths. However, anatomically the reflex path length for the quadriceps femoris muscle is shorter than for the gastrocnemius muscle, as is the distance from the common flexor muscles of the forearm at the elbow than for the gastrocnemius muscle at the ankle. Both muscle groups consistently demonstrated faster frequencies, in the same patient, when compared to the frequency of the gastrocnemius muscle at the ankle. Furthermore, the measurements to the anterior superior iliac spine would be an under-estimate of the distance, rather than an over-estimate compared to the upper limb measurements.

It is concluded that clonus frequency in spastic muscle is a direct consequence of reflex path length and this finding confirms the theory of a peripheral, rather than a central mechanism for clonus.

\section{References}

1 Gottlieb GL, Agarwal GC. Physiological clonus in man. Exp Neurol 1977;54:616-21.

${ }^{2}$ Dimitrijevic MR, Nathan PW, Sherwood AM. Clonus: The Role of Central Mechanisms. J Neurol Neurosurg Psychiatry 1980;43:321-33.

${ }^{3}$ Szumski AJ, Burg D, Struppler A, Vehlo F. Activity of muscle spindles during muscle twitch and clonus in normal and spastic human subjects. Electroencephalogr Clin Neurophysiol 1974;37:589-97.

${ }^{4}$ Hagbarth KE, Wallin G, Lofstedt L, Aquilonius SM. Muscle spindle activity in alternating tremor of Parkinsonism and clonus. J Neurol Neurosurg Psychiatry 1975;38:636-41.

5 Walsh EG. Clonus: Beats provoked by the application of a rhythmic force. J Neurol Neurosurg Psychiatry 1976;39:266-74.

${ }^{6}$ Stein RB, Lee RG. Tremor and Clonus. In: Brooks VB, ed. Handbook of Physiology Section 1: Vol II. The Nervous System. Baltimore: Williams \& Wilkins 1981:325-43. 